\title{
Counting to one: reducibility of one- and two-loop amplitudes at the integrand level
}

\author{
Ronald H.P. Kleiss, ${ }^{a}$ loannis Malamos, ${ }^{b, 1}$ Costas G. Papadopoulos ${ }^{c, d}$ and Rob \\ Verheyen $^{a}$ \\ ${ }^{a}$ Radboud University, \\ Nijmegen, The Netherlands \\ ${ }^{b}$ Instituto de Física Corpuscular, \\ Consejo Superior de Investigaciones Científicas-Universitat de València, \\ Parc Cientific, E-46980 Paterna (Valencia), Spain. \\ 'NCSR 'Demokritos', Agia Paraskevi, \\ 15310, Greece \\ ${ }^{d}$ Department of Physics, Theory Division, CERN, \\ 1211 Geneva 23, Switzerland \\ E-mail: R.Kleiss@science.ru.nl, Ioannis.Malamos@ific.uv.es, \\ costas.papadopoulos@cern.ch, robverheyen@gmail.com
}

ABSTRACT: Calculation of amplitudes in perturbative quantum field theory involve large loop integrals. The complexity of those integrals, in combination with the large number of Feynman diagrams, make the calculations very difficult. Reduction methods proved to be very helpful, lowering the number of integrals that need to be actually calculated. Especially reduction at the integrand level improves the speed and set-up of these calculations. In this article we demonstrate, by counting the numbers of tensor structures and independent coefficients, how to write such relations at the integrand level for one- and two-loop amplitudes. We clarify their connection to the so-called spurious terms at one loop and discuss their structure in the two-loop case. This method is also applicable to higher loops, and the results obtained apply to both planar and non-planar diagrams.

KEYwords: QCD Phenomenology, NLO Computations

ArXiv EPRINT: 1206.4180

\footnotetext{
${ }^{1}$ Supported by REA Grant Agreement PITN-GA-2010-264564 (LHCPhenoNet), by the MICINN Grants FPA2007-60323, FPA2011-23778 and Consolider-Ingenio 2010 Programme CSD2007-00042 (CPAN).
} 


\section{Contents}

1 Introduction 1

2 Reduction at one loop 3

2.1 Introduction: reduction with trivial coefficients 3

2.2 Reduction with coefficients linear in the loop momentum 5

3 Reduction of two-loop integrands $\quad 9$

3.1 Preliminaries 9

$\begin{array}{lll}3.2 & \text { Further reduction with linear terms } & 11\end{array}$

$\begin{array}{lll}3.3 & \text { Comments on the number of independent coefficients } & 12\end{array}$

$\begin{array}{lll}3.4 & \text { Reduction with general quadratic terms } & 14\end{array}$

$\begin{array}{ll}3.5 & \text { Reduction with general cubic terms } \\ \end{array}$

$\begin{array}{lll}4 & \text { Conclusions } & 17\end{array}$

$\begin{array}{ll}\text { A Pentagons to boxes with the OPP method } & 18\end{array}$

\section{Introduction}

Modern colliders such as the Large Hadron Collider (LHC) (and the Tevatron before it was shut down) produce a large amount of experimental data. In order to understand the output of these experiments, comparison between very precise theoretical results and experimental results is needed. It is clear, from the theoretical point of view, that Nextto-Leading-Order (NLO) and Next-to-Next-to-Leading-Order (NNLO) calculations with many external legs have to be considered [1]. This implies that (very) large loop integrals have to be computed for very many Feynman diagrams, which has widely been considered the bottleneck of such calculations.

Reduction techniques form a way out. The idea of reducing Feynman integrals with a large number of denominators to a set of simpler integrals (i.e. with fewer denominators) at one loop goes surprisingly many years back [2,3]. A typical integral with $n$ such denominators, in $d$ space-time dimensions, is given by

$$
\int d^{d} q \frac{1}{D_{1} D_{2} \ldots D_{n}}
$$

where $D_{i}=\left(q+p_{i}\right)^{2}-m_{i}^{2}$ is the denominator of a generic propagator. In [2] the authors reduce a triangle (integral with 3 denominators) to bubbles (2 denominators) in 2 dimensions while in [3] a pentagon ( 5 denominators) is reduced to boxes (4 denominators) in 4 dimensions. We see that the result of the reduction depends on the dimension. However, 
the methods we will use can be applied to all dimensions. Our main interest is of course the case $d=4$.

Since a few decades now [4], it is well known fact a generic one-loop amplitude is decomposable in terms of scalar integrals, with one, two, three and four external legs (in $d=4)$. Passarino and Veltman [5] used Lorentz invariance to express tensor one-loop $n$-point integrals in terms of $m$-point scalar integrals $(m \leq n)$. As a consequence, only the evaluation of scalar integrals (integrals with trivial numerators) is needed in order to perform a one-loop calculation.

In another attempt [6] a pentagon-to-boxes decomposition is performed in 4 dimensions. The importance of this paper is that it provides a basis in four-dimensional momentum space (the so called van Neerven-Vermaseren basis), which proved useful for understanding one-loop reduction. Another important fact about this paper is the use of what we call nowadays spurious terms to decompose a scalar pentagon to boxes. Spurious terms are terms that, by construction, vanish upon integration. Their rôle will be explained later when we consider reductions at the integrand level and we shall see why one cannot avoid them.

The next big step comes from unitarity methods [7-11]. Instead of working with specific Feynman diagrams these methods have a big advantage in that they try to decompose the whole one-loop amplitude in terms of the scalar integrals. By cutting propagators ${ }^{1}$ the rational coefficients of loop integrals are given in terms of products of tree amplitudes. In generalized unitarity methods [12-17], the notion of multiple cuts is introduced. One can cut more than one propagator to find these coefficients. Note that, for $d=4$, cutting four propagators essentially determines the loop momentum (there is, in general, more than a single solution since the $D$ 's are quadratic in the loop momentum).

The Ossola-Papadopoulos-Pittau (OPP) method [18-22] comes as a natural combination of all the above. Since every integral can be decomposed to scalar integrals with up to four denominators (for $d=4$ ), every one-loop amplitude is written in terms of coefficients that multiply these scalar integrals. The OPP method works at the integrand level [23, 24], which means that for these decompositions to be possible one must also include spurious terms. Then one has to find a way to calculate the coefficients of the reduction and multiply them with the appropriate scalar integrals, using one of the packages available for the evaluation of them(i.e. [25, 26]). Finding the coefficients is a purely algebraic problem. The method is suitable for a fully numerical implementation. The OPP method has been widely used so far in many one loop calculations (see for example [27-41]).

As we noticed before, in the case of one-loop calculations a basis for any integral is known in advance. Any one-loop integral can be written in terms of scalar boxes, triangles, bubbles and tadpoles. However, in the case of higher-loop integrals the situation is different. A basis is not known a priori. It is believed that unitarity methods can also be applied in that case and there are some recent papers in that direction, performing decomposition à la OPP [42-45], or using generalized unitarity [46-48] at two loops.

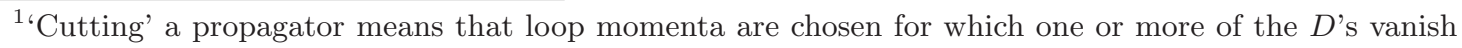
so that the integrand becomes singular. One also speaks of 'putting propagators on-shell'.
} 
Two remarks are in order here. The first is that the basis of two-loop integrals does not include only scalar integrals. It includes integrals that also have irreducible scalar products (ISP) as numerators (to some power) that cannot be rewritten as existing denominators of the integral. In the one-loop case these ISP are always spurious and integrate to zero, but for higher loops this does no longer hold. The second remark is that if one is interested in constructing a unitarity-like basis, the set of integrals that ends up with is not necessarily a minimal one: the integrals are not by default Master Integrals (MI). There might be smaller sets of true MI and at two or more loops one can find them by using integration-by-parts (IBP) identities [49-51].

In this article we prove that any two-loop integral can be written as a linear combination of integrals with at most $2 d$ denominators. From this set of integrals, one can in principle end up with true master integrals with numerators containing ISP 's. The layout of this paper is the following. We start with definitions and reduction at one loop. We see why one can have unitarity-like bases at the integrand level by writing first the numerator of our integrals (for scalar cases the number one) as a sum of coefficients times denominators. We investigate, using simple algebra, what is the form of these coefficients in order for our polynomial equation to have solutions. Then we repeat the same procedure in the case of two-loop integrands. Our method is not exactly the OPP one, but the connection of the two methods will become clear.

\section{Reduction at one loop}

\subsection{Introduction: reduction with trivial coefficients}

We start with integrands of any given one-loop amplitude. These integrands consist of the sum of integrands coming from the Feynman diagrams that contribute to a given process and share the same topology; the advantage is that we perform the decomposition once instead of reducing every single diagram separately. For that reason we deal with integrand-graphs, or iGraphs instead of Feynman diagrams.

We define the $i G r a p h$ as a collection of denominators with arbitrary momenta. We use the freedom of shifting in every loop momentum.

The order of the iGraph is simply the number of propagators it includes. We give an example of an iGraph of order 5 (pentagon) below, where $j=1 \ldots 5$,

$$
D_{j} \equiv D\left(q+p_{j}\right)=\left(q+p_{j}\right)^{2}-m_{j}{ }^{2}=q^{2}+2\left(p_{j} \cdot q\right)+\mu_{j} \quad, \quad \mu_{j} \equiv p_{j}{ }^{2}-m_{j}{ }^{2},
$$

The loop momentum is denoted by $q^{\mu}$, and $p_{j}{ }^{\mu}$ is called the external momentum, where it must be realized that by this we do not mean a momentum related to a particle incoming or outgoing in a given amplitude: what we call external momenta are simply fixed momenta, given in some way by the configuration of incoming and outgoing momenta and the various diagram topologies.

We deal only with scalar iGraphs, that have unity for their numerator. Let us regard an iGraph of order $n$ with a nontrivial numerator, for instance

$$
\frac{q \cdot k}{D_{1} D_{2} \cdots D_{N}} \text {. }
$$




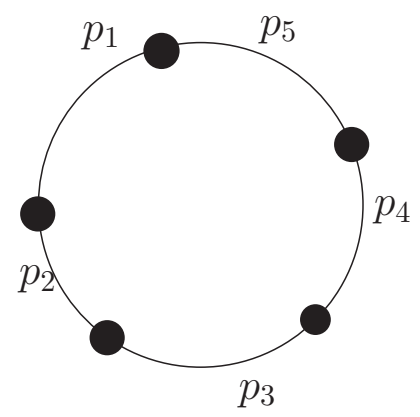

Figure 1. A one-loop pentagon iGraph. The dots serve to distinguish the several denominators. The external momenta are indicated.

Now, we can always arrange for $p_{1}{ }^{\mu}=0$ by a suitable shift of the loop momentum, and write the vector $k^{\mu}$ as

$$
k^{\mu}=\omega^{\mu}+2 \sum_{j=2}^{n} \zeta_{j} p_{j}{ }^{\mu},
$$

where the $\zeta$ 's are fixed numbers and $\omega \cdot p_{j}=0$ for all $j$ (if $n \geq d+1$ then $\omega^{\mu}$ simply vanishes). We can then write

$$
(q \cdot k)=(q \cdot \omega)+\sum_{j=2}^{n} \zeta_{j}\left(D_{j}-D_{1}-\mu_{j}+\mu_{1}\right),
$$

so that this nonscalar iGraph decomposes into scalar iGraphs of order $n$ and $n-1$, plus possibly a spurious term about which we do not worry since it integrates to zero. Our treatment of the scalar case is therefore sufficiently general.

Consider a one-loop iGraph of order $n$ :

$$
\frac{1}{D_{1} D_{2} D_{3} D_{4} \ldots D_{n}} \text {. }
$$

We say that we can decompose this iGraph if we can find funtions $T_{1,2, \ldots, n}(q)$ such that

$$
D_{1} T_{1}(q)+D_{2} T_{2}(q)+\cdots+D_{n} T_{n}(q)=1
$$

for then we have

$$
\frac{1}{D_{1} \cdots D_{n}}=\frac{T_{1}(q)}{D_{2} D_{3} D_{4} \ldots D_{n}}+\frac{T_{2}(q)}{D_{1} D_{3} D_{4} \ldots D_{n}}+\cdots+\frac{T_{n}(q)}{D_{1} D_{2} D_{3} \ldots D_{n-1}},
$$

and the original iGraph is decomposed into a sum of iGraphs of order $n-1$ (or lower).

This immediately leads us to state the following theorem: one-loop iGraphs of order $d$ or smaller cannot be decomposed in the above manner. The reason is simple: for $n \leq d$ there exist a cut through all propagators, so that $D_{j}=0$ for $j=1, \ldots, n$ and eq. (2.4) then would become $0=1$.

The simplest possibility for the functions $T_{j}(q)$ is to take them to be just numbers independent of $q^{\mu}$ ('trivial' coefficients):

$$
T_{j}(q)=x_{j} .
$$


From eq. (2.4) then we have

$$
q^{2} \sum_{j=1}^{n} x_{j}+2 q_{\mu} \sum_{j=1}^{n} x_{j} p_{j}{ }^{\mu}+\sum_{j=1}^{n} x_{j} \mu_{j}=1 .
$$

Since this has to hold for any value of $q^{\mu}$ we must have separately

$$
\sum_{j=1}^{n} x_{j}=0 \quad, \quad \sum_{j=1}^{n} x_{j} p_{j}{ }^{\mu}=0
$$

and

$$
\sum_{j=1}^{n} x_{j} \mu_{j}=1
$$

Note that if a nontrivial solution to the homogeneous equations (2.7) exists, then by suitable scaling we can always satisfy eq. (2.8). We see that, at one loop, for $d=4$ any iGraph of order 6 or higher can be decomposed in this fairly trivial way. In this case, we have a system of 6 equations with 6 or more unknowns that we can solve. A pentagon in 4-dimensions thus cannot be decomposed that way. For general $d$, iGraphs of order $d+2$ or higher are decomposable with trivial coefficients since for $n \geq d+2$, a solution to the system of the $d+2$ eq. (2.7) and eq. (2.8) can always be found.

\subsection{Reduction with coefficients linear in the loop momentum}

For a one-loop iGraph of order 5 (or lower) no trivial decomposition exists in $d=4$, assuming that four out of the five external momenta, $p_{j}$, in the loop can be considered as linearly independent. One way to see this is by shifting the loop momentum so that $\sum_{j} p_{j}{ }^{\mu}=0$. Then the only solution to the conditions in eq. (2.7) is $x_{j}=0, j=1 \ldots, 5$ which is unacceptable in eq. (2.8). We therefore turn to the next simplest possibility for the $T$ 's, with a linear $q$ dependence:

$$
T_{j}(q)=x_{j}+\sum_{k=1}^{4} x_{j, k}\left(q \cdot t_{k}\right) .
$$

The single $x_{j}$ is now replaced by 5 (or $d+1$ ) variables to be determined in each $T$. $^{2}$

Here, the four (or $d$ ) vectors $t_{k}{ }^{\mu}$ must be linearly independent but are otherwise arbitrary. In analogy to eq. (2.7) and eq. (2.8), we now have more tensor structures in terms of the loop momentum: we can denote them by the shorthand

$$
1, q^{\mu}, q^{\mu} q^{\nu}, q^{2} q^{\mu}
$$

There are, for $d=4$, therefore $1+4+10+4=19$ independent tensor structures. It is easy to see why only these tensor structures appear. The linear dependence of the coefficients times the $q^{2}$ part of the propagators give rise to the $q^{2} q^{\mu}$ terms, which are the terms of the

\footnotetext{
${ }^{2}$ In the $d$ dimensional case, the sum in eq. (2.9) runs from 1 to $d$ instead of 4 because $d$ vectors are needed for a basis. We shall work in general dimension, focusing of course in the case $d=4$.
} 


\begin{tabular}{|r|ccccc|}
\hline$k$ & 0 & 1 & 2 & 3 & 4 \\
\hline$d=1$ & 3 & 4 & 5 & 6 & 7 \\
2 & 4 & 8 & 13 & 19 & 26 \\
3 & 5 & 13 & 26 & 45 & 71 \\
4 & 6 & 19 & 45 & 90 & 161 \\
5 & 7 & 26 & 71 & 161 & 322 \\
6 & 8 & 34 & 105 & 266 & 588 \\
\hline
\end{tabular}

Table 1. Values of $N(d, k)$.

highest rank. The other terms produce all lower tensor structures up to constant terms coming from the constant part of the coeffients times the constant part of the propagators. Note that the $q^{2}$ term that could be produced from the quadratic part of a propagator times the constant part of the coefficients is no longer independent and it is included as the trace part of $q^{\mu} q^{\nu}$. This can be extended to the inclusion of higher-rank tensors and other dimensions: in $d$ dimensions, and with the inclusion of tensor up to rank $k$, we find for the number $N(d, k)$ of independent tensor structures

$$
N(d, k)=\left(\begin{array}{c}
d-1+k \\
k
\end{array}\right)+\sum_{p=0}^{k+1}\left(\begin{array}{c}
d-1+p \\
p
\end{array}\right) .
$$

In table 1 we give the results for various ranks and dimensionalities.

The number of coefficients $x$ to be solved for is given by

$$
X(n, d, k)=n\left(\begin{array}{c}
d+k \\
k
\end{array}\right) .
$$

Since for $d=4$ and $k=1$ we have $N(4,1)=19$, it would seem that iGraphs of order 5 and 4 are decomposable with linear terms. However, the situation is not so simple since it is not obvious that the 25 coefficients for $n=5$ and the 20 coefficients for $n=4$ allow us to actually build up the 19 required tensor structures. We now describe how we can ascertain the number of independent structures numerically, by an approach that may be dubbed cancellation probing.

We start by generating random values for the external momenta $p_{j}{ }^{\mu}$ and $m_{j}(j=$ $1, \ldots, n)$. This avoids any possibility of us choosing, coincidentally, any special phase space point where degeneracy might occur. Then, we choose random values for $q^{\mu}$ precisely $\xi=X(n, d, k)$ times, and insert all this in eq. (2.4). We are left with a set of $\xi$ linear equations for the $\xi$ unknowns $x$ :

$$
\sum_{j=1}^{\xi} M^{i}{ }_{j} x^{j}=1 \quad, \quad j=1, \ldots, \xi
$$

The $\xi \times \xi$ matrix $M$ is purely numerical. We obtain it using the computer-algebra package MAPLE $^{3}$ which, although not numerically the fastest available, has the essential advantage

\footnotetext{
${ }^{3}$ http://www . maplesoft.com/.
} 


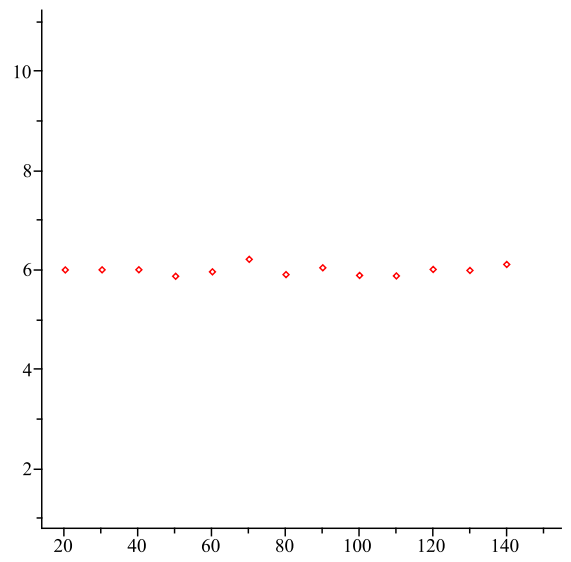

Figure 2. The number of zero eigenvalues using rounding errors for the case of a pentagon decomposition with coefficients linear in the loop momentum. The obtained value is $q=5.988 \pm 0.098$; the (absolute values of the $25 \times 25$ determinants range from $1.610^{-826}$ to $2.310^{-108}$.

that one can easily set the precision with which numerical operations are performed. ${ }^{4}$ Now, if the number of independent tensor structures that can be formed with our $T$ 's is less than $\xi$, the determinant of $M$ will vanish. In an ideal real-number model of computation, we would thus find $\operatorname{det}(M)=0$, but in our actual numerical computation there will be rounding errors. A cancellation of numbers to 'zero' will, in MAPLE, actually give a number of order $10^{-p}$, where $p$ is the number of digits specifies in the precision we tell MAPLE to use. If the matrix' determinant is computed by Gaussian elimination, ${ }^{5}$ then a matrix with $q$ zero eigenvalues will have a determinant of order $10^{-p q}$. By letting $p$ run down from 150 to 20 in steps of 10 , we can obtain ${ }^{6}$ a very accurate estimate of $q$, especially since $q$ must be integer. We give two examples to demonstrate the use of this method for the calculation of the zero eigenvalues. In both examples we consider a decomposition of pentagon to boxes, with linear and quartic terms respectively.

The difference $\xi-q$ then gives the rank of $M$, and this determines the decomposability of the iGraph: the rank must be equal to $N(d, k)$ for it to be decomposable. Note that the rank can be in general equal (for soluble cases) or smaller (insoluble cases) than the number of tensor structures. It can never be larger than the tensor structures since one cannot construct more tensor structures than actually exist. In table 2 we give the results of cancellation probing for various $n$ and $d$.

We have denoted the limit of decomposability with horizontal lines. We conclude that in four dimensions, $n=5$ is precisely decomposable, but $n=4$ is not. We now also see the deeper reason for this: in spite of there being 20 coefficients (one more than the minimum of 19), only 17 independent combinations can actually be formed. We also see that for sufficiently large $n$ the number of independent combinations of coefficients saturates at

\footnotetext{
${ }^{4}$ The relevant variable is Digits.

${ }^{5}$ This is almost unavoidable since the matrix $M$ is not sparse, and anyway we can choose Gaussian elimination as an option in any case.

${ }^{6}$ Surprisingly, the cancellation probing appears to fail for $p=15$ and $p=10$, possibly since MAPLE may have special ways to treat these accuracies ( $p=10$ is default in MAPLE).
} 


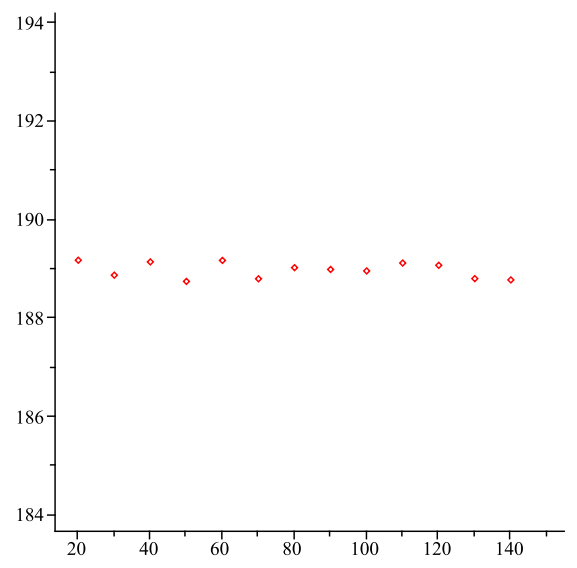

Figure 3. The number of zero eigenvalues using rounding errors for the case of a pentagon decomposition with up to quartic in the loop momentum coefficients. Here, $q=188.98 \pm 0.15$, with the $350 \times 350$ determinants ranging from $2.710^{-26000}$ to $2.310^{-3320}$.

\begin{tabular}{|c|c|c|c|c|c|c|}
\hline$n$ & $d=6$ & $d=5$ & $d=4$ & $d=3$ & $d=2$ & $d=1$ \\
\hline 2 & $14-0$ & $12-0$ & $10-0$ & $8-0$ & $6-0$ & $4-0$ \\
3 & $21-1$ & $18-1$ & $15-1$ & $12-1$ & $9-1$ & $6-2$ \\
4 & $28-3$ & $24-3$ & $20-3$ & $16-3$ & $12-4$ & $8-4$ \\
5 & $35-6$ & $30-6$ & $25-6$ & $20-7$ & $15-7$ & $10-6$ \\
6 & $42-10$ & $36-10$ & $30-11$ & $24-11$ & $18-10$ & $12-8$ \\
7 & $49-15$ & $42-16$ & $35-16$ & $28-15$ & $21-13$ & $14-10$ \\
8 & $56-22$ & $48-22$ & $40-21$ & $32-19$ & $24-16$ & $16-12$ \\
\hline$N(d, 1)$ & 34 & 26 & 19 & 13 & 8 & 4 \\
\hline
\end{tabular}

Table 2. The rank of $M$ for various $d$ and $n$, given as the difference $\xi-q$.

$N(d, 1)$ as it ought to. We conclude that in $d$ dimensions, an iGraph of order $d+1$ is precisely decomposable with linear terms, but one of order $d$ is of course not.

In the OPP method [18], the linear terms are precisely the spurious terms. We have to note that our general linear terms are not exactly those. The spurious terms have a specific property leading to fewer tensor structures, and we give an example in the appendix. Rewriting our general linear terms in terms of propagators and spurious terms, we see that we decompose a pentagon into boxes and triangles (like in [6] and [18] for example). It can be checked that the triangles always cancel, and therefore the decomposition is actually unique.

At this point it must be pointed out that, in all cases where a decomposition is possible in principle, we actually have obtained a solution for, the system (2.13). Once a would-be solution is found, it can easily be tested by evaluating eq. (2.4) for additional random values of the loop momentum ${ }^{7}$ This 'global $1=1$ test' then verifies this solution, where of course the equality is supposed to hold only up to the precision used.

\footnotetext{
${ }^{7}$ In a certain sense, eq. (2.4) implies an infinite number of linear equations, of which we take $\xi$ and solve them.
} 


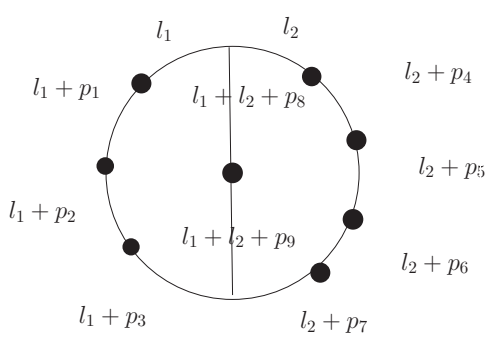

Figure 4. An example of the two-loop iGraph $(4,2,5)$. One can see the three different loop lines.

\section{Reduction of two-loop integrands}

\subsection{Preliminaries}

We now turn to the problem of reducibility at two loops. Recently several attempts in this direction have appeared in the literature (see i.e. $[42,43,46]$ ). Let us assume that $l_{1}$ and $l_{2}$ are the two loop momenta. We consider three different kind of propagators for the three different loop lines of a generic two loop iGraph.

$$
D\left(l_{1}+p_{i}\right), D\left(l_{2}+p_{j}\right), D\left(l_{1}+l_{2}+p_{k}\right),
$$

where for instance $D\left(l_{i}+p_{j}\right)=\left(l_{i}+p_{j}\right)^{2}-m_{j}^{2}$ and the $p_{j}$ are the external momenta associated with the propagators of the diagram.

Such iGraphs can be denoted by the triplet $\left(n_{1}, n_{2}, n_{3}\right)$ which indicates the number $n_{1}$ of propagators that contain only the one loop momentum $l_{1}$, the number $n_{3}$ of propagators containing only the other loop momentum $l_{2}$, and the $n_{2}$ propagators containing both. Obviously due to the symmetries of the iGraphs, for instance exchange $l_{1} \leftrightarrow l_{2}$, we have relations of the form $\left(n_{1}, n_{2}, n_{3}\right) \leftrightarrow\left(n_{3}, n_{2}, n_{1}\right)$ or $\left(n_{1}, n_{2}, n_{3}\right) \leftrightarrow\left(n_{1}, n_{3}, n_{2}\right)$ provided we also exchange properly the external momenta. Predictably, we write the total order of the iGraph as $n=n_{1}+n_{2}+n_{3}$.

The propagators depending on both loop momenta are called mixed propagators. If these are absent the two integrals factor out and the problem becomes a double copy of one loop integrals. The same happens in case any other loop line is missing since, by shifting, one can always arrange the loop momenta such that they factor out. We consider these cases solved (by the one loop techniques) and will not discuss them further.

The general strategy consists in finding function $x_{j}$ satisfying the following equation

$$
\begin{aligned}
& \sum_{j=1}^{n_{1}} x_{j}\left(l_{1}, l_{2}\right) D\left(l_{1}+p_{j}\right)+\sum_{j=n_{1}+1}^{n_{1}+n_{2}} x_{j}\left(l_{1}, l_{2}\right) D\left(l_{1}+l_{2}+p_{j}\right)+ \\
& \sum_{j=n_{1}+n_{2}+1}^{n} x_{j}\left(l_{1}, l_{2}\right) D\left(l_{2}+p_{j}\right)=1 .
\end{aligned}
$$

As in the one-loop case, a generic graph of order $n \leq 2 d$ with $n_{1,2,3} \leq 4$ cannot be decomposed in this way, since there are $l_{1,2}$ momenta for which all propagators appearing in the above equation can be simultaneously on-shell. This does not imply on the 
other hand that iGraphs of higher order must always be decomposable for any phase-space and mass configuration. A counterexample is the Feynman diagram of order 5 in two dimensions: ${ }^{8}$ if all internal lines in this self-energy Feynman diagram are massless, it is possible to choose the two loop momenta components such that all five propagators are simultaneously on-shell.

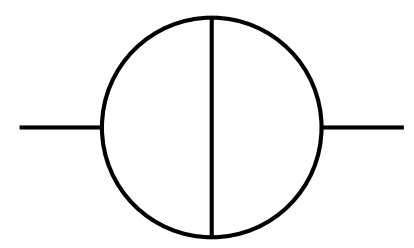

The requirement for trivial decomposition (for $x_{j}$ that are independent of $l_{1}$ and $l_{2}$ in eq. (3.2)) now reads

$$
\begin{aligned}
\sum_{j=1}^{n_{1}+n_{2}} x_{j} & =\sum_{j=n_{1}+1}^{n} x_{j}=\sum_{j=n_{1}+1}^{n_{1}+n_{2}} x_{j}=0, \\
\sum_{j=1}^{n_{1}+n_{2}} x_{j} p_{j}{ }^{\mu} & =\sum_{j=n_{1}+1}^{n} x_{j} p_{j}{ }^{\mu}=0,
\end{aligned}
$$

and

$$
\sum_{j=1}^{n} x_{j} \mu_{j}=1 .
$$

In total there are $2 d+4$ conditions, so that the minimum size of a trivially decomposable iGraph is $2 d+4$. In four dimensions, scalar iGraphs can therefore be decomposed down to $n=11$. Again in analogy, for $n=11$, since by shifting we can arrange $p_{1}+\cdots+p_{n_{1}+n_{2}}=0$ as well as $p_{n_{1}+1}+\cdots+p_{n}=0$, the only solution to the $2 d+3$ homogeneous equations is $x_{j}=0, j=1, \ldots, n$, and this fails the inhomogeneous equation. On the other hand, since any subset of an iGraph is itself an iGraph, any iGraph with $n_{1} \geq 6, n_{2} \geq 6$, or $n_{3} \geq 6$ is trivially decomposable (for $d=4$ ). Furthermore, with linear terms we see, from the one-loop discussion ${ }^{9}$ that we only have to consider two-loop iGraphs with

$$
n_{1,2,3} \leq 4(=d), \quad n_{1}+n_{2}+n_{3} \leq 11(=2 d+3) .
$$

A word of caution is in order here. We may have a case where $n_{1}+n_{2} \geq 6$ or $n_{2}+n_{3} \geq 6$ and then decide to perform a decomposition à la one-loop with trivial terms, taking $l_{1}$ as the loop momentum, for instance, and $l_{2}$ as one of the "external" momenta or vice-versa. Since the solution of the eq. (3.2) is nonlinear with respect to the external momenta, due to

\footnotetext{
${ }^{8}$ This diagram can, in fact, be decomposed, but not by the method described above: instead one has to use IBP techniques.

${ }^{9}$ In case there at least 6 propagators in one loop line we can first reduce the propagators in this loop line with constant coefficients and then continue further if possible. In the case of 5 propagators we already know that adding linear terms that depend only on the loop momentum of this loop line and take all coefficients that depend on the other loop momentum to zero, we can again solve the problem à la one loop.
} 
matrix inversion, the resultant decomposition will not have, in general, the simple form of iGraphs again, and the emerging integrals will belong to very different classes of functions.

The number of two-loop iGraphs that we have to consider is therefore not very large: 4 for $d=2,10$ for $d=3,19$ for $d=4$.

\section{2 $\quad$ Further reduction with linear terms}

With trivial decomposition we see that we can always end up with an iGraph of order $2 d+3$. Like in the one loop case, we now add coefficients linear in the loop momentum and hope for further reductions.

A note is in order here. In the one loop case the resulting integrals were always scalar. The reason is that any contraction of the loop momentum with any vector can either reconstruct denominators or be a spurious term. After integrating, in the case a denominator is reconstructed the remaining integral is a scalar integral with fewer denominators. In case the term is spurious it vanishes after integration. In two loops this is not the case anymore. One can always use dot products of the loop momenta with the momenta of the integrals to write relations like

$$
2 l_{1} \cdot p_{j}=\left[\left(l_{1}+p_{j}\right)^{2}-m_{j}^{2}\right]-l_{1}^{2}-p_{j}^{2}+m_{j}^{2} .
$$

The denominator $D\left(l_{1}+p_{j}\right)$ may, however, not be present in the integral in case $p_{j}$ appears in a propagator of another type such as $D\left(l_{2}+p_{j}\right)^{2}$. Then, the product $l_{1} \cdot p_{j}$ may be an irreducible scalar product (ISP) [42]. But not always; the ISP's of an integral are more complicated to write. For example, if there are enough propagators of the type $\left(l_{1}+p_{i}\right)^{2}$ in the diagram such that the $p_{i}$ 's can form a basis, one can rewrite $p_{j}$ as a linear combination of the $p_{i}$ 's and manage to reconstruct denominators. There is a specific number of ISP's in any diagram and one can have some freedom in how to write them. The integrals of the resulting basis can have numerators with ISP's in some power. It is not obvious that a scalar integral with a specific number of denominators is more difficult to calculate than a non-scalar integral with fewer denominators; however it is commonly accepted that this is the case.

Contrary to the one-loop case, scalar integrals are not sufficient to express any two-loop integral. However, as far as reducibility is concerned, studying scalar integrals is sufficient since if the scalar integral is decomposable, then decomposability holds for the non scalar one with the same denominators.

Again, we want to write, if possible, the number 1 as in eq. (3.2). We use general linear terms in the sense that in every dimension we construct a basis $t_{i}$ (possibly, but not necessarily, the external momenta in the iGraph) and we have

$$
x_{i}=a_{i}+\sum_{j} b_{i j}\left(l_{1} \cdot t_{j}\right)+\sum_{j} c_{i j}\left(l_{2} \cdot t_{j}\right)
$$

with the $a_{i}, b_{i j}$, and $c_{i j}$ constants with respect to the loop momenta. Since in $d$ dimensions, we need $d$ vectors to construct such a basis, it is obvious that for an iGraph of order $n$ we start with $(2 d+1) n$ coefficients. As in the one-loop case, we give a table that contains, for 


\begin{tabular}{|c|c|c|c|c|c|c|}
\multicolumn{8}{c|}{$T(d)=2 d^{2}+9 d+1$} \\
\hline$n$ & $d=6$ & $d=5$ & $d=4$ & $d=3$ & $d=2$ & $d=1$ \\
\hline 3 & $39-0$ & $33-0$ & $27-0$ & $21-0$ & $15-0$ & $9-0$ \\
\hline 4 & $52-0$ & $44-0$ & $36-0$ & $28-0$ & $20-0$ & $12-2$ \\
5 & $65-1$ & $55-1$ & $45-1$ & $35-1$ & $25-1$ & $15-5$ \\
6 & $78-3$ & $66-3$ & $54-3$ & $42-3$ & $30-3$ & \\
7 & $91-6$ & $77-6$ & $63-6$ & $49-6$ & $35-8$ & \\
8 & $104-10$ & $88-10$ & $72-10$ & $56-10$ & & \\
9 & $111-15$ & $99-15$ & $81-15$ & $63-17$ & & \\
10 & $130-21$ & $110-21$ & $90-21$ & & & \\
11 & $143-28$ & $121-28$ & $99-30$ & & & \\
12 & $156-36$ & $132-36$ & & & & \\
13 & $169-45$ & $143-47$ & & & & \\
14 & $182-55$ & & & & & \\
15 & $195-55$ & & & & & \\
\cline { 2 - 2 }$T(d)$ & 127 & 96 & 69 & 46 & 27 & 10 \\
\hline
\end{tabular}

Table 3. As in table 2 for linear terms.

every dimension we worked with, the number of tensor structures (denoted by $T(d)$ ) and the number of independent coefficients that we have explicitly calculated by cancellation probing as described above.

In table 3 a line distinguishes between reducible and non-reducible cases. Reducibility is explicitly checked by eq. (3.2). For all iGraphs defined by eq. (3.6), we see that the number of independent coefficients becomes equal to the number of independent tensor structures, when reducibility is attained. In all dimensions every $n=2 d+2$ case is reducible with linear terms to a $n=2 d+1$ iGraph. In four dimensions, we can decompose every integral down to integrals with 9 denominators. At this point, we are one step away from the limiting value of 8 .

\subsection{Comments on the number of independent coefficients}

The most difficult part in our counting is always the number of independent coefficients. As shown, we do it numerically but we would like to understand more the reason why we have as many independent coefficients as we do find. We try to demonstrate here a way to estimate this number; for the case of linear terms we will give some examples.

We can rewrite the terms in eq. (3.8). As we mentioned, terms of the form $l_{i} \cdot p_{j}$ either reconstruct denominators either become ISP. Let us assume the $(4,1,4)$ iGraph in four dimensions. It has in principle two ISP that we call $\sigma_{1}$ and $\sigma_{2}$. To see this, we note that in any of the loop lines that consists of four denominators, there are three external momenta. We can always "borrow" a fourth one from the other line to have a complete basis and write any product $l_{i} \cdot p_{j}$ as a linear combination of the four propagators and an ISP. The ISP in that case would be the product of $l_{i}$ with the momentum we borrowed. Repeating for the other loop line we get the two ISP's. Using the coefficients of eq. (3.8) 
in eq. (3.2) we can either get a denominator times a constant or an ISP, or products of two denominators. We write this equation schematically as

$$
1=\sum_{i=1}^{9} D_{i}\left\{1, \sigma_{1}, \sigma_{2}\right\}+\sum_{i=1}^{9} \sum_{j, j \geq i}^{9} D_{i} D_{j}\{1\}
$$

This means that by writing $\left\{1, \sigma_{1}, \sigma_{2}\right\} D_{i}$ there is a constant coefficient in front of every of these different terms:

$$
\left\{1, \sigma_{1}, \sigma_{2}\right\}=a \cdot 1+b_{1} \sigma_{1}+b_{2} \sigma_{2} .
$$

where a, $b_{1}, b_{2}$ general numbers. In our particular example, we have $9 \times 3+45 \times 1=72$ coefficients. However, we started with a problem with up to cubic power in loop momenta and ended with up to quartic powers since we have these products of two denominators times some constants. These higher powers have to cancel, which means that we have to put extra constraints on our coefficients. We have 6 such constraints to cancel, namely the

$$
l_{1}^{4}, l_{1}^{2} l_{2}^{2}, l_{2}^{4},\left(l_{1} \cdot l_{2}\right) l_{1}^{2},\left(l_{1} \cdot l_{2}\right) l_{2}^{2},\left(l_{1} \cdot l_{2}\right)^{2}
$$

terms. As a result we end up with $72-6=66$ independent coefficients, which is the number we get numerically as well. If we now try to decompose an iGraph of order 10 we can prove that eq. (3.9) becomes

$$
1=\sum_{i=1}^{10} D_{i}\left(1, \sigma_{1}, \sigma_{2}\right)+\sum_{i=1}^{9} \sum_{j, j \geq i}^{9} D_{i} D_{j}(1) .
$$

We don't need to go up to 10 in the product of 2 denominators since ${ }^{10}$

$$
D_{10} \propto\left(D_{1}, \ldots, D_{9}, \sigma_{1}, \sigma_{2}\right) .
$$

We still need them, though, in the first term to produce terms of the type $\left(\sigma_{i}\right)^{2}$. In that case we have 69 independent coefficients and this graph is reducible. Adding more propagators we do not get more independent coefficients. In the same way one can count the independent coefficients in all dimensions although it is clear that it is safer to find their number numerically since there are a lot of overlaps in the tensor structures for higher cases. The way of rewriting the general linear terms as propagators and ISP's in the example above is still not the OPP method. In an extension of the OPP method to two loops, one would find the ISP's of every subdiagram and would avoid terms like $D_{i}^{2}$. We expect something similar to the one-loop case to happen then, rewriting $\sigma_{1}$ and $\sigma_{2}$ in the form of true ISP's of every subdiagram's contributions of the terms with the highest number of denominators to cancel. For that it is possible that special properties exist, as again in the one-loop case where spurious term solve a lot of equations by putting automatically tensor structures to zero.

\footnotetext{
${ }^{10}$ This is actually the point where the number of dimensions plays a role in the counting.
} 


\subsection{Reduction with general quadratic terms}

We try to reduce our iGraphs further with the use of general quadratic terms in the coefficients. In this case the coefficients become

$$
\begin{aligned}
x_{i}= & a_{i}+\sum_{j} b_{i j}\left(l_{1} \cdot t_{j}\right)+\sum_{j} c_{i j}\left(l_{2} \cdot t_{j}\right)+\sum_{j \leq k} d_{i j k}\left(l_{1} \cdot t_{j}\right)\left(l_{1} \cdot t_{k}\right) \\
& \left.+\sum_{j \leq k} e_{i j k}\left(l_{2} \cdot t_{j}\right)\left(l_{2} \cdot t_{k}\right)+\sum_{j, k} f_{i j k}\left(l_{1} \cdot t_{j}\right)\left(l_{2} \cdot t_{k}\right)\right) .
\end{aligned}
$$

We give the number of tensor structures $T(d)$ and the original number of coefficients with general quadratic terms, $C_{1}(d)$. The coefficients depend on the number of propagators $n$. Notice that the expression for $T(d)$ is not valid for $d=2$. In that case, there is more overlap between the highest tensor structures. More specifically, for this particular case one can completely reconstruct the $l_{1}^{2} l_{2}^{\mu} l_{2}^{\nu}$ structure from $l_{2}^{2} l_{1}^{\mu} l_{1}^{\nu}$ and $\left(l_{1} \cdot l_{2}\right) l_{1}^{\mu} l_{2}^{\nu}$ allowing fewer independent structures to be constructed.

$$
\begin{aligned}
T(d)= & 4 d^{3} / 3+10 d^{2}+20 d / 3-2 \\
= & 8\left(\begin{array}{c}
d \\
3
\end{array}\right)+28\left(\begin{array}{c}
d \\
2
\end{array}\right)+18\left(\begin{array}{l}
d \\
1
\end{array}\right)-2 \\
& C_{1}(d)=\left(2 d^{2}+3 d+1\right) n .
\end{aligned}
$$

The second form for $T(d)$ shows that it always evalutes to integers for integer $d$. With quadratic terms we start with $\left(2 d^{2}+3 d+1\right) \times n$ coefficients in total, not all of them of course being independent. Using cancellation probing we are able to find the number of independent coefficients for different iGraphs in different dimensions. We put our findings in table 4 .

In table 4 a line distinguishes again between reducible and non-reducible cases, according to eq. (3.2). As we see, we can decompose in 2 dimensions an iGraph of order 5 to lower order iGraphs as indicated by unitarity. However, there is no such solution in 3 and 4 dimensions and in this case we have to investigate what happens if we add cubic, quartic terms and so on. The two-dimensional case is exceptional here because of the extra properties that lower the number of tensor structures.

\subsection{Reduction with general cubic terms}

We focus now on 3 and 4 dimensions since we finished the reduction in 2 dimensions. We include general cubic terms and our coefficients become

$$
\begin{aligned}
x_{i}= & a_{i}+\sum_{j} b_{i j}\left(l_{1} \cdot t_{j}\right)+\sum_{j} c_{i j}\left(l_{2} \cdot t_{j}\right)+\sum_{j \leq k} d_{i j k}\left(l_{1} \cdot t_{j}\right)\left(l_{1} \cdot t_{k}\right) \\
& +\sum_{j \leq k} e_{i j k}\left(l_{2} \cdot t_{j}\right)\left(l_{2} \cdot t_{k}\right)+\sum_{j, k} f_{i j k}\left(l_{1} \cdot t_{j}\right)\left(l_{2} \cdot t_{k}\right) \\
& +\sum_{j \leq k \leq l} g_{i j k l}\left(l_{1} \cdot t_{j}\right)\left(l_{1} \cdot t_{k}\right)\left(l_{1} \cdot t_{l}\right)+\sum_{l} \sum_{j \leq k} h_{i j k l}\left(l_{1} \cdot t_{j}\right)\left(l_{1} \cdot t_{k}\right)\left(l_{2} \cdot t_{l}\right) \\
& \left.+\sum_{l} \sum_{j \leq k} p_{i j k l}\left(l_{2} \cdot t_{j}\right)\left(l_{2} \cdot t_{k}\right)\left(l_{1} \cdot t_{l}\right)+\sum_{j \leq k \leq l} q_{i j k l}\left(l_{2} \cdot t_{j}\right)\left(l_{2} \cdot t_{k}\right)\left(l_{2} \cdot t_{l}\right)\right) .
\end{aligned}
$$




\begin{tabular}{|c|c|c|c|c|c|}
\hline$n$ & $d=6$ & $d=5$ & $d=4$ & $d=3$ & $d=2$ \\
\hline 3 & $273-3$ & $198-3$ & $135-4$ & $84-3$ & $45-3$ \\
4 & $364-6$ & $264-6$ & $180-6$ & $128-6$ & $60-6$ \\
\cline { 5 - 6 } 5 & $455-22$ & $330-20$ & $225-18$ & $140-16$ & $75-15$ \\
6 & $546-50$ & $396-44$ & $270-38$ & $168-32$ & $90-30$ \\
7 & $637-89$ & $462-77$ & $315-65$ & $196-53$ & \\
8 & $728-138$ & $528-118$ & $360-98$ & $224-80$ & \\
9 & $819-196$ & $594-166$ & $405-136$ & $252-108$ & \\
10 & $910-262$ & $660-220$ & $450-180$ & & \\
11 & $1001-335$ & $726-279$ & $495-225$ & & \\
12 & $1092-414$ & $792-344$ & $540-270$ & & \\
13 & $1183-498$ & $858-410$ & & & \\
\cline { 2 - 2 } 14 & $1274-588$ & & & & \\
15 & $1365-679$ & & & & \\
\cline { 1 - 2 }$T(d)$ & 686 & 448 & 270 & 144 & 60 \\
\hline
\end{tabular}

Table 4. As in table 2 for quadratic terms.

We give the number of tensor structures $T(d)$ and the original number of coefficients with general cubic terms $C_{1}(d)$. The coefficients depend on the number of propagators $n$. The expression for $T(d)$ is valid for $d \geq 3$.

$$
\begin{aligned}
T(d)= & 2 d^{4} / 3+22 d^{3} / 3+71 d^{2} / 6+d / 6+1= \\
& 16\left(\begin{array}{l}
d \\
4
\end{array}\right)+68\left(\begin{array}{l}
d \\
3
\end{array}\right)+77\left(\begin{array}{l}
d \\
2
\end{array}\right)+20\left(\begin{array}{l}
d \\
1
\end{array}\right)+1 \\
C_{1}(d)= & \left(4 d^{3} / 3+4 d^{2}+11 d / 3+1\right) n=8\left(\begin{array}{l}
d \\
3
\end{array}\right)+16\left(\begin{array}{l}
d \\
2
\end{array}\right) 9\left(\begin{array}{l}
d \\
1
\end{array}\right)+1 .
\end{aligned}
$$

In $d$ dimensions we start with $C_{1}(d)$ coefficients, not all of them being independent. We run the MAPLE code in the case of the iGraph of order 7 in 3 dimensions and we find that out of 588 original coefficients, 360 are independent. This is the number of tensor structures as well. Using another PYTHON-based program, ${ }^{11}$ we can actually solve the system decomposing any iGraph of order 7 in 3 dimensions to lower iGraphs with general cubic terms, and perform the $1=1$ test. This means that with cubic terms we are able to decompose any two-loop iGraph in 3 dimensions to up to a $2 d$ iGraph as expected from unitarity. In the same way, we can investigate $d=4$, and we get a valid decomposition: from our original 1485 coefficients, 831 are independent and all the tensor structures can be reconstructed. Actually we did the same in 5, 6, 7, and 8 dimensions, and managed to decompose every integral of generic order $2 d+1$ or higher, using cubic terms. We believe that this is a general result for any dimension, except of course for $d=2$, where the same can be achieved with only quadratic terms. We put our findings, up to dimension 6 , in table 5 .

\footnotetext{
${ }^{11}$ http://codepad.org/zT4wUxCJ .
} 


\begin{tabular}{|c|c|c|c|c|c|}
\multicolumn{7}{c}{$T(d)=2 d^{4} / 3+22 d^{3} / 3+71 d^{2} / 6+d / 6+1$} \\
\hline$n$ & $d=6$ & $d=5$ & $d=4$ & $d=3$ & $d=2$ \\
\hline 3 & & & & & $105-15$ \\
4 & & & & & $140-32$ \\
5 & & & & $420-88$ & $175-61$ \\
6 & & & & $504-152$ & $210-96$ \\
7 & & & $1155-352$ & $588-228$ & \\
8 & & & $1320-497$ & $672-312$ & \\
9 & & $2574-971$ & $1485-654$ & & \\
10 & & $2860-1237$ & $1650-819$ & & \\
11 & $5005-2157$ & $3146-1515$ & & & \\
12 & $5460-2592$ & $3432-1801$ & & & \\
\cline { 3 - 6 } 13 & $5915-3039$ & & & & \\
14 & $6370-3494$ & & & & \\
\hline$T(d)$ & 2876 & 1631 & 831 & 360 & 114 \\
\hline
\end{tabular}

Table 5. As in table 2 for cubic terms.

In table 5, the horizontal line distinguishes again between reducible and non-reducible cases.

With this table we finish our analysis for the reducibility of one- and two-loop amplitudes at the integrand level. Before we move to our conclusions, two notes are in order. Firstly, there may be specific phase-space points for which such a decomposition is not allowed. An example of such a phase-space point could be one such that for specific values of the loop momenta, more than $d$ in one loop and $2 d$ at two loops propagators can be set to zero simultaneously. That would lead to problems since for that values of the loop momenta the equation $1=1$ is not satisfied. However, we do not consider such configurations. We are interested in general cases (that was the reason to deal with iGraphs in the first place). In our experience, in these special phase-space points, the integrals are still reducible via IBP identities.

Secondly, in section 2.1 we have a theorem stating that any one-loop iGraph of order $d$ or smaller (and similarly any two-loop iGraph of order $2 d$ or smaller with $n_{1,2,3} \leq d$ ) cannot be decomposed in the way we proposed. As an extra check of our numerical method, we included coefficients of even higher rank (quadratic, cubic for the one loop case and quartic for the two-loop case) and as it was expected, no further decomposition was achieved. That is an extra proof that our numerical method is robust.

Summarising our findings, we give table 6 that describes the reducibility of the iGraphs we investigated. The horizontal line is to distinguish the reducible from the non-reducible cases in all dimensions. As we described, the iGraphs of order higher than $2 d$ are always reducible, while the ones with less or equal to $2 d$ are not. We specify, in the cases the iGraph is reducible, the smallest rank for which the reduction can be achieved. $C U, Q, L$ and co stand for cubic, quadratic, linear and constant terms respectively. 


\begin{tabular}{|c|c|c|c|c|c|}
\hline$n$ & $d=6$ & $d=5$ & $d=4$ & $d=3$ & $d=2$ \\
\hline 3 & & & & & NOT \\
4 & & & & & NOT \\
5 & & & & NOT & $Q$ \\
6 & & & & NOT & $L$ \\
7 & & & NOT & $C U$ & $L$ \\
8 & & & NOT & $L$ & $c o$ \\
9 & & NOT & $C U$ & $L$ & $c o$ \\
10 & & NOT & $L$ & $c o$ & $\cdot$ \\
11 & NOT & $C U$ & $L$ & $c o$ & $\cdot$ \\
12 & NOT & $L$ & $c o$ & $\cdot$ & $\cdot$ \\
13 & $C U$ & $L$ & $c o$ & $\cdot$ & \\
14 & $L$ & $c o$ & $\cdot$ & $\cdot$ & \\
15 & $L$ & $\cdot$ & $\cdot$ & & \\
16 & $c o$ & $\cdot$ & $\cdot$ & & \\
\hline \hline
\end{tabular}

Table 6. Reducibility of iGraphs. CU, Q, L and co stand for cubic, quadratic, linear and constant terms respectively.

\section{Conclusions}

By introducing the notion of iGraphs, we have investigated the decomposability of Feynman amplitudes at one and two loops. In both cases we have demonstrated (in the two-loop case, up to $d=8$ ) that generic iGraphs can be decomposed down to the unitarity-based limit of $n=d$ and $n=2 d$, respectively. For one-loop graphs, the inclusion of linear terms is sufficient, and we have elucidated the relation between these linear terms and the OPP's spurious terms. At the two-loop level, ultimately cubic terms are needed (quadratic for $d=2$ ). If one wants to design a two-loop OPP method, it is clear that one has to take our general linear, quadratic, cubic terms and rewrite them in terms of propagators (this would lead to contributions with less denominators) and ISP's of each subdiagram seperately ${ }^{12}$ leading to scalar and non scalar integrals, where the latter might or might not be spurious (see i.e. [43]).

Our work is basically the starting point of an OPP method and a proof that reductions that were conjectured in $[42,43]$ are actually valid and survive the global $1=1$ test.

We note once again that the resulting integral basis, obtained in this way, is clearly not a minimal one. We are aware of cases that could be further decomposed using IBP identities. Achieving a proper level of understanding of the interplay between OPP and IBP will certainly open the road for an efficient reduction of two-loop amplitudes at the integrand level.

\footnotetext{
${ }^{12}$ In the same way that one-loop OPP has different spurious terms for every subdiagram.
} 


\section{Acknowledgments}

We acknowledge useful discussions with Prof. M. Czakon.

\section{A Pentagons to boxes with the OPP method}

By defining

$$
D_{n}\left(p_{1}, p_{2}, \ldots, p_{n}\right)=\frac{1}{D\left(q+p_{1}\right) D\left(q+p_{2}\right) \ldots D\left(q+p_{n}\right)},
$$

we try to decompose this pentagon

$$
D_{5}\left(p_{1}, p_{2}, p_{3}, p_{4}, p_{5}\right)
$$

to the 5 following boxes

$$
\begin{gathered}
D_{4}\left(p_{2}, p_{3}, p_{4}, p_{5}\right), D_{4}\left(p_{3}, p_{4}, p_{5}, p_{1}\right), D_{4}\left(p_{4}, p_{5}, p_{1}, p_{2}\right) \\
D_{4}\left(p_{5}, p_{1}, p_{2}, p_{3}\right) D_{4}\left(p_{1}, p_{2}, p_{3}, p_{4}\right) .
\end{gathered}
$$

By shifting the loop momenta we can always arrange that the momenta $p_{i}$ of the denominators sum up to zero:

$$
\sum_{i=1}^{5} p_{i}=0 .
$$

We write the spurious terms in the following form

$$
\begin{aligned}
S_{1}= & \epsilon\left(q+p_{2}, q+p_{3}, q+p_{4}, q+p_{5}\right)=q_{\mu}\left(\epsilon^{\mu}\left(p_{3}, p_{4}, p_{5}\right)-\epsilon^{\mu}\left(p_{2}, p_{4}, p_{5}\right)+\right. \\
& \left.\epsilon^{\mu}\left(p_{2}, p_{3}, p_{5}\right)-\epsilon^{\mu}\left(p_{2}, p_{3}, p_{4}\right)\right)+\epsilon\left(p_{2}, p_{3}, p_{4}, p_{5}\right)= \\
& q_{\mu} A_{1}^{\mu}+B_{1} .
\end{aligned}
$$

We then have:

$$
\begin{aligned}
& A_{1}^{\mu}=\epsilon^{\mu}\left(p_{3}, p_{4}, p_{5}\right)-\epsilon^{\mu}\left(p_{2}, p_{4}, p_{5}\right)+\epsilon^{\mu}\left(p_{2}, p_{3}, p_{5}\right)-\epsilon^{\mu}\left(p_{2}, p_{3}, p_{4}\right), \\
& A_{2}^{\mu}=\epsilon^{\mu}\left(p_{4}, p_{5}, p_{1}\right)-\epsilon^{\mu}\left(p_{3}, p_{5}, p_{1}\right)+\epsilon^{\mu}\left(p_{3}, p_{4}, p_{1}\right)-\epsilon^{\mu}\left(p_{3}, p_{4}, p_{5}\right), \\
& A_{3}^{\mu}=\epsilon^{\mu}\left(p_{5}, p_{1}, p_{2}\right)-\epsilon^{\mu}\left(p_{4}, p_{1}, p_{2}\right)+\epsilon^{\mu}\left(p_{4}, p_{5}, p_{2}\right)-\epsilon^{\mu}\left(p_{4}, p_{5}, p_{1}\right), \\
& A_{4}^{\mu}=\epsilon^{\mu}\left(p_{1}, p_{2}, p_{3}\right)-\epsilon^{\mu}\left(p_{5}, p_{2}, p_{3}\right)+\epsilon^{\mu}\left(p_{5}, p_{1}, p_{3}\right)-\epsilon^{\mu}\left(p_{5}, p_{1}, p_{2}\right), \\
& A_{5}^{\mu}=\epsilon^{\mu}\left(p_{2}, p_{3}, p_{4}\right)-\epsilon^{\mu}\left(p_{1}, p_{3}, p_{4}\right)+\epsilon^{\mu}\left(p_{1}, p_{2}, p_{4}\right)-\epsilon^{\mu}\left(p_{1}, p_{2}, p_{3}\right) .
\end{aligned}
$$

Using the OPP master formula [18] we get

$$
\begin{aligned}
1= & {\left[d_{1}+\tilde{d}_{1} S_{1}\right] D\left(q+p_{1}\right)+\left[d_{2}+\tilde{d}_{2} S_{2}\right] D\left(q+p_{2}\right)+\ldots+} \\
& {\left[d_{5}+\tilde{d}_{5} S_{5}\right] D\left(q+p_{5}\right) . }
\end{aligned}
$$

We compare the polynomials of the two sides of eq. (A.4). The $q^{2} q_{\mu}$ terms must vanish for any value of $q$ which means

$$
\sum_{i}\left(\tilde{d}_{i} A_{i}^{\mu}\right)=0
$$


Notice that from eq. (A.3)

$$
\sum_{i=1}^{5} A_{i}=0
$$

which means that if all $\tilde{d}_{i}$ are equal eq. (A.5) is satisfied.

This is actually the only solution. To see that, consider the sum

$$
\tilde{d}_{1} A_{1}^{\mu}+\cdots+\tilde{d}_{5} A_{5}^{\mu}
$$

Substituting

$$
p_{5}=-p_{1}-p_{2}-p_{3}-p_{4}
$$

we get

$$
\begin{aligned}
\sum\left(\tilde{d}_{i} A_{i}^{\mu}\right)= & \left(\tilde{d}_{1}+\tilde{d}_{2}-4 \tilde{d}_{3}+\tilde{d}_{4}+\tilde{d}_{5}\right) \epsilon^{\mu}\left(p_{1}, p_{2}, p_{4}\right) \\
& +\left(-\tilde{d}_{1}+4 \tilde{d}_{2}-\tilde{d}_{3}-\tilde{d}_{4}-\tilde{d}_{5}\right) \epsilon^{\mu}\left(p_{1}, p_{3}, p_{4}\right) \\
& +\left(-\tilde{d}_{1}-\tilde{d}_{2}-\tilde{d}_{3}+4 \tilde{d}_{4}-\tilde{d}_{5}\right) \epsilon^{\mu}\left(p_{1}, p_{2}, p_{3}\right) \\
& +\left(-4 \tilde{d}_{1}+\tilde{d}_{2}+\tilde{d}_{3}+\tilde{d}_{4}+\tilde{d}_{5}\right) \epsilon^{\mu}\left(p_{2}, p_{3}, p_{4}\right)
\end{aligned}
$$

In 4 dimensions, for this sum to be zero, all coefficients in front of the vectors are zero (the vectors are linearly independent). This results in the system:

$$
\begin{aligned}
\tilde{d}_{1}+\tilde{d}_{2}-4 \tilde{d}_{3}+\tilde{d}_{4}+\tilde{d}_{5} & =0, \\
-\tilde{d}_{1}+4 \tilde{d}_{2}-\tilde{d}_{3}-\tilde{d}_{4}-\tilde{d}_{5} & =0, \\
-\tilde{d}_{1}-\tilde{d}_{2}-\tilde{d}_{3}+4 \tilde{d}_{4}-\tilde{d}_{5} & =0, \\
-4 \tilde{d}_{1}+\tilde{d}_{2}+\tilde{d}_{3}+\tilde{d}_{4}+\tilde{d}_{5} & =0 .
\end{aligned}
$$

One can solve the system and find

$$
\tilde{d}_{1}=\tilde{d}_{2}=\tilde{d}_{3}=\tilde{d}_{4}=\tilde{d}_{5}
$$

The only solution is when all coefficients are equal and from now on we call them $\tilde{d}$. Then we take a look at the quadratic in $q$ parts of the right-hand side of eq. (A.4). They cancel as well but one has to be careful since they come from two terms, the $q^{2}$ and the $q^{\mu} q^{\nu}$ term. We look at the latter term

$$
\tilde{d} q_{\mu} \sum_{i=1}^{5}\left(q \cdot p_{i}\right) A_{i}^{\mu} .
$$

We have:

$$
\begin{aligned}
\tilde{d} q_{\mu} \sum_{i=1}^{5}\left(q \cdot p_{i}\right) A_{i}^{\mu}= & \tilde{d} q_{\mu}\left(\left(A_{1}-A_{5}\right)\left(q \cdot p_{1}\right)+\right. \\
& \left.\left(A_{2}-A_{5}\right)\left(q \cdot p_{2}\right)+\left(A_{3}-A_{5}\right)\left(q \cdot p_{3}\right)+\left(A_{4}-A_{5}\right)\left(q \cdot p_{4}\right)\right),
\end{aligned}
$$


where $p_{5}=-p_{1}-p_{2}-p_{3}-p_{4}$ is again used. With this substitution we have:

$$
\begin{aligned}
& A_{1}-A_{5}=-5 \epsilon^{\mu}\left(p_{2}, p_{3}, p_{4}\right), \\
& A_{2}-A_{5}=5 \epsilon^{\mu}\left(p_{1}, p_{3}, p_{4}\right), \\
& A_{3}-A_{5}=-5 \epsilon^{\mu}\left(p_{1}, p_{2}, p_{4}\right), \\
& A_{4}-A_{5}=5 \epsilon^{\mu}\left(p_{1}, p_{2}, p_{3}\right) .
\end{aligned}
$$

Inserting the Schouten Identity

$$
\begin{aligned}
\epsilon\left(p_{1}, p_{2}, p_{3}, p_{4}\right) q^{\mu}= & \epsilon^{\mu}\left(p_{2}, p_{3}, p_{4}\right)\left(q \cdot p_{1}\right)-\epsilon^{\mu}\left(p_{1}, p_{3}, p_{4}\right)\left(q \cdot p_{2}\right)+ \\
& \epsilon^{\mu}\left(p_{1}, p_{2}, p_{4}\right)\left(q \cdot p_{3}\right)-\epsilon^{\mu}\left(p_{1}, p_{2}, p_{3}\right)\left(q \cdot p_{4}\right),
\end{aligned}
$$

we get

$$
\tilde{d} q_{\mu} \sum_{i=1}^{5}\left(q \cdot p_{i}\right) A_{i}^{\mu}=-5 \tilde{d} q^{2} \epsilon\left(p_{1}, p_{2}, p_{3}, p_{4}\right)
$$

That is exactly the property that the spurious terms have that makes the solution to the system possible. The $q^{\mu} q^{\nu}$ terms all vanish owing to the Schouten identity except for the trace part proportional to $q^{2}$ solving 9 out of 10 equations in one go. The total number of nontrivial equations is therefore 10 and not $19 \mathrm{in}$ this case and the system has a solution. To complete the story, taking into account eq. (A.8) we are left with $5 d_{i}$ and one $\tilde{d}$, which are uniquely now determined from the remaining 6 equations, namely constant part, $q^{2}$ term and $q_{\mu}$ term.

Open Access. This article is distributed under the terms of the Creative Commons Attribution License which permits any use, distribution and reproduction in any medium, provided the original author(s) and source are credited.

\section{References}

[1] B. Anastasiou, Standard model theory for collider physics, PoS EPS-HEP2009 (2009) 007.

[2] G. Kallen and J. Toll, Special class of Feynman integrals in two-dimensional space-time, J. Math. Phys. 6 (1965) 299 [InSPIRE].

[3] D. Melrose, Reduction of Feynman diagrams, Nuovo Cim. 40 (1965) 181 [inSPIRE].

[4] G. 't Hooft and M. Veltman, Scalar one loop integrals, Nucl. Phys. B 153 (1979) 365 [INSPIRE].

[5] G. Passarino and M. Veltman, One loop corrections for $e^{+} e^{-}$annihilation into $\mu^{+} \mu^{-}$in the Weinberg model, Nucl. Phys. B 160 (1979) 151 [InSPIRE].

[6] W. van Neerven and J. Vermaseren, Large loop integrals, Phys. Lett. B 137 (1984) 241 [INSPIRE].

[7] Z. Bern, L.J. Dixon, D.C. Dunbar and D.A. Kosower, One loop $N$ point gauge theory amplitudes, unitarity and collinear limits, Nucl. Phys. B 425 (1994) 217 [hep-ph/9403226] [INSPIRE]. 
[8] Z. Bern, L.J. Dixon, D.C. Dunbar and D.A. Kosower, Fusing gauge theory tree amplitudes into loop amplitudes, Nucl. Phys. B 435 (1995) 59 [hep-ph/9409265] [INSPIRE].

[9] Z. Bern, L.J. Dixon and D.A. Kosower, On-shell recurrence relations for one-loop QCD amplitudes, Phys. Rev. D 71 (2005) 105013 [hep-th/0501240] [INSPIRE].

[10] Z. Bern, L.J. Dixon and D.A. Kosower, The last of the finite loop amplitudes in QCD, Phys. Rev. D 72 (2005) 125003 [hep-ph/0505055] [inSPIRE].

[11] Z. Bern, L.J. Dixon and D.A. Kosower, Bootstrapping multi-parton loop amplitudes in QCD, Phys. Rev. D 73 (2006) 065013 [hep-ph/0507005] [inSPIRE].

[12] R. Britto, F. Cachazo and B. Feng, Generalized unitarity and one-loop amplitudes in $N=4$ super-Yang-Mills, Nucl. Phys. B 725 (2005) 275 [hep-th/0412103] [INSPIRE].

[13] R. Britto, F. Cachazo, B. Feng and E. Witten, Direct proof of tree-level recursion relation in Yang-Mills theory, Phys. Rev. Lett. 94 (2005) 181602 [hep-th/0501052] [INSPIRE].

[14] R. Britto, F. Cachazo and B. Feng, New recursion relations for tree amplitudes of gluons, Nucl. Phys. B 715 (2005) 499 [hep-th/0412308] [INSPIRE].

[15] C. Anastasiou, R. Britto, B. Feng, Z. Kunszt and P. Mastrolia, D-dimensional unitarity cut method, Phys. Lett. B 645 (2007) 213 [hep-ph/0609191] [INSPIRE].

[16] C.F. Berger, Z. Bern, L.J. Dixon, D. Forde and D.A. Kosower, On-shell unitarity bootstrap for QCD amplitudes, Nucl. Phys. Proc. Suppl. 160 (2006) 261 [hep-ph/0610089] [InSPIRE].

[17] W.T. Giele, Z. Kunszt and K. Melnikov, Full one-loop amplitudes from tree amplitudes, JHEP 04 (2008) 049 [arXiv: 0801.2237] [INSPIRE].

[18] G. Ossola, C.G. Papadopoulos and R. Pittau, Reducing full one-loop amplitudes to scalar integrals at the integrand level, Nucl. Phys. B 763 (2007) 147 [hep-ph/0609007] [INSPIRE].

[19] G. Ossola, C.G. Papadopoulos and R. Pittau, Numerical evaluation of six-photon amplitudes, JHEP 07 (2007) 085 [arXiv:0704.1271] [INSPIRE].

[20] G. Ossola, C.G. Papadopoulos and R. Pittau, CutTools: a program implementing the OPP reduction method to compute one-loop amplitudes, JHEP 03 (2008) 042 [arXiv:0711.3596] [INSPIRE].

[21] G. Ossola, C.G. Papadopoulos and R. Pittau, On the rational terms of the one-loop amplitudes, JHEP 05 (2008) 004 [arXiv:0802.1876] [INSPIRE].

[22] P. Mastrolia, G. Ossola, C. Papadopoulos and R. Pittau, Optimizing the reduction of one-loop amplitudes, JHEP 06 (2008) 030 [arXiv:0803.3964] [INSPIRE].

[23] D. Forde, Direct extraction of one-loop integral coefficients, Phys. Rev. D 75 (2007) 125019 [arXiv: 0704.1835] [INSPIRE].

[24] R.K. Ellis, Z. Kunszt, K. Melnikov and G. Zanderighi, One-loop calculations in quantum field theory: from Feynman diagrams to unitarity cuts, Phys. Rept. 518 (2012) 141 [arXiv: 1105.4319] [INSPIRE].

[25] A. van Hameren, OneLOop: for the evaluation of one-loop scalar functions, Comput. Phys. Commun. 182 (2011) 2427 [arXiv:1007.4716] [INSPIRE].

[26] R.K. Ellis and G. Zanderighi, Scalar one-loop integrals for QCD, JHEP 02 (2008) 002 [arXiv:0712.1851] [INSPIRE]. 
[27] T. Binoth, G. Ossola, C. Papadopoulos and R. Pittau, NLO QCD corrections to tri-boson production, JHEP 06 (2008) 082 [arXiv:0804.0350] [INSPIRE].

[28] A. van Hameren, C. Papadopoulos and R. Pittau, Automated one-loop calculations: a proof of concept, JHEP 09 (2009) 106 [arXiv:0903.4665] [INSPIRE].

[29] G. Bevilacqua, M. Czakon, C. Papadopoulos, R. Pittau and M. Worek, Assault on the NLO wishlist: $p p \rightarrow t \bar{t} b \bar{b}$, JHEP 09 (2009) 109 [arXiv: 0907.4723] [INSPIRE].

[30] G. Bevilacqua, M. Czakon, C. Papadopoulos and M. Worek, Dominant QCD backgrounds in Higgs boson analyses at the LHC: a study of $p p \rightarrow t \bar{t}+2$ jets at next-to-leading order, Phys. Rev. Lett. 104 (2010) 162002 [arXiv:1002.4009] [INSPIRE].

[31] G. Bevilacqua, M. Czakon, A. van Hameren, C.G. Papadopoulos and M. Worek, Complete off-shell effects in top quark pair hadroproduction with leptonic decay at next-to-leading order, JHEP 02 (2011) 083 [arXiv: 1012.4230] [INSPIRE].

[32] A. Kardos, C. Papadopoulos and Z. Trócsányi, Top quark pair production in association with a jet with NLO parton showering, Phys. Lett. B 705 (2011) 76 [arXiv:1101.2672] [INSPIRE].

[33] G. Bevilacqua, M. Czakon, C. Papadopoulos and M. Worek, Hadronic top-quark pair production in association with two jets at next-to-leading order $Q C D$, Phys. Rev. D 84 (2011) 114017 [arXiv:1108.2851] [inSPIRE].

[34] A. Kardos, Z. Trócsányi and C. Papadopoulos, Top quark pair production in association with a Z-boson at NLO accuracy, Phys. Rev. D 85 (2012) 054015 [arXiv:1111.0610] [INSPIRE].

[35] M. Garzelli, A. Kardos, C. Papadopoulos and Z. Trócsányi, Z0 - boson production in association with a top anti-top pair at NLO accuracy with parton shower effects, Phys. Rev. D 85 (2012) 074022 [arXiv:1111.1444] [INSPIRE].

[36] V. Hirschi, R. Frederix, S. Frixione, M.V. Garzelli, F. Maltoni, et al., Automation of one-loop QCD corrections, JHEP 05 (2011) 044 [arXiv:1103.0621] [INSPIRE].

[37] R. Frederix, S. Frixione, V. Hirschi, F. Maltoni, R. Pittau, et al., $W$ and $Z / \gamma *$ boson production in association with a bottom-antibottom pair, JHEP 09 (2011) 061 [arXiv:1106.6019] [INSPIRE].

[38] R. Frederix, S. Frixione, V. Hirschi, F. Maltoni, R. Pittau, et al., Four-lepton production at hadron colliders: aMC@NLO predictions with theoretical uncertainties, JHEP 02 (2012) 099 [arXiv: 1110.4738] [INSPIRE].

[39] R. Frederix, S. Frixione, V. Hirschi, F. Maltoni, R. Pittau, et al., aMC@NLO predictions for Wjj production at the Tevatron, JHEP 02 (2012) 048 [arXiv:1110.5502] [INSPIRE].

[40] M. Worek, On the next-to-leading order QCD K-factor for top anti-top bottom anti-bottom production at the Tevatron, JHEP 02 (2012) 043 [arXiv:1112.4325] [INSPIRE].

[41] G. Bevilacqua and M. Worek, Constraining BSM physics at the LHC: four top final states with NLO accuracy in perturbative QCD, JHEP 07 (2012) 111 [arXiv:1206.3064] [INSPIRE].

[42] P. Mastrolia and G. Ossola, On the integrand-reduction method for two-loop scattering amplitudes, JHEP 11 (2011) 014 [arXiv:1107.6041] [INSPIRE].

[43] S. Badger, H. Frellesvig and Y. Zhang, Hepta-cuts of two-loop scattering amplitudes, JHEP 04 (2012) 055 [arXiv: 1202.2019] [INSPIRE].

[44] Y. Zhang, Integrand-level reduction of loop amplitudes by computational algebraic geometry methods, JHEP 09 (2012) 042 [arXiv: 1205.5707] [INSPIRE]. 
[45] P. Mastrolia, E. Mirabella, G. Ossola and T. Peraro, Scattering amplitudes from multivariate polynomial division, Phys. Lett. B $\mathbf{7 1 8}$ (2012) 173 [arXiv:1205.7087] [INSPIRE].

[46] J. Gluza, K. Kajda and D.A. Kosower, Towards a basis for planar two-loop integrals, Phys. Rev. D 83 (2011) 045012 [arXiv: 1009.0472] [INSPIRE].

[47] D.A. Kosower and K.J. Larsen, Maximal unitarity at two loops, Phys. Rev. D 85 (2012) 045017 [arXiv: 1108.1180] [INSPIRE].

[48] R.M. Schabinger, A new algorithm for the generation of unitarity-compatible integration by parts relations, JHEP 01 (2012) 077 [arXiv:1111.4220] [INSPIRE].

[49] F. Tkachov, A theorem on analytical calculability of four loop renormalization group functions, Phys. Lett. B 100 (1981) 65 [INSPIRE].

[50] K. Chetyrkin and F. Tkachov, Integration by parts: the algorithm to calculate $\beta$-functions in 4 loops, Nucl. Phys. B 192 (1981) 159 [inSPIRE].

[51] V.A. Smirnov, Evaluating Feynman integrals, Springer Tracts Mod. Phys. 211 (2004) 1. 